\title{
Spontaneous Symmetry Breaking in the U(2) Planar Thirring Model?
}

\author{
Simon Hands ${ }^{* \dagger}$ \\ Department of Physics, College of Science, \\ Swansea University, Swansea SA2 8PP, United Kingdom. \\ E-mail: s.hands@swan.ac.uk
}

\begin{abstract}
Whether the $\mathrm{U}(2 N)$ symmetry of Dirac fermions in $2+1$ space-time dimensions is spontaneously broken by pair condensation once interactions are present is an important problem in nonperturbative quantum field theory. Here I focus on the Thirring model, whose interaction is a current-current contact term, using numerical simulations of a lattice model formulated with domain wall fermions - it has previously been demonstrated that $\mathrm{U}(2 N)$ symmetry is recovered in the limit of infinite wall separation. I present results obtained with flavor numbers $N=1$ and 2 , and will attempt to put both upper and lower bounds on $N_{c}$, the critical number of flavors above which symmetry breaking does not occur even for arbitrarily strong coupling. The resulting $N_{c}$ will be shown to be very far from the value $N_{c} \approx 6.6$ obtained using staggered lattice fermions, which do not observe $\mathrm{U}(2 N)$ symmetry.
\end{abstract}

XIII Quark Confinement and the Hadron Spectrum - Confinement 2018

31 July - 6 August 2018

Maynooth University, Ireland

\footnotetext{
*Speaker.

${ }^{\dagger}$ Acknowledges financial support from STFC and the Leverhulme Trust.
} 


\section{Introduction}

Relativistic fermions moving in $2+1$ spacetime dimensions form the basis of some facscinating quantum field theories, whose principal applications mainly fall in the domain of the condensed matter physics of layered systems. For example, fermion degrees of freedom described by a Dirac equation have been invoked and studied in models of the underdoped regime of $d$-wave superconductors $[1,2]$, where they arise as excitations at the nodes of the gap function $\Delta(\vec{k})$; in models of spin-liquid behaviour in Heisenberg antiferromagnets [3, 4], where the infrared behaviour of compact $\mathrm{QED}_{3}$ remains an open issue; as surface states of three-dimensional topological insulators; and of course as low-energy electronic excitations in graphene [5].

What all these models share in common is their use of four-component reducible spinor representations for the fermi fields. For non-interacting fermions the action in Euclidian metric is

$$
S=\int d^{3} x \bar{\psi}\left(\gamma_{\mu} \partial_{\mu}\right) \psi+m \bar{\psi} \psi ; \mu=0,1,2
$$

a key point is the mass term proportional to $m$ is hermitian and invariant under the parity inversion $x_{\mu} \mapsto-x_{\mu}$. For $m=0 S$ has a global U(2) invariance generated by

$$
\begin{gathered}
\psi \mapsto e^{i \alpha} \psi, \bar{\psi} \mapsto \bar{\psi} e^{-i \alpha} ; \quad \psi \mapsto e^{\alpha \gamma_{3} \gamma_{5}} \psi, \bar{\psi} \mapsto \bar{\psi} e^{-\alpha \gamma_{3} \gamma_{5}} ; \\
\psi \mapsto e^{i \alpha \gamma_{3}} \psi, \bar{\psi} \mapsto \bar{\psi} e^{i \alpha \gamma_{3}} ; \quad \psi \mapsto e^{i \alpha \gamma_{5}} \psi, \bar{\psi} \mapsto \bar{\psi} e^{i \alpha \gamma_{5}}
\end{gathered}
$$

For $m \neq 0$ the $\gamma_{3}$ and $\gamma_{5}$ rotations (1.3) are no longer symmetries; the general pattern of breaking is thus $\mathrm{U}(2 N) \rightarrow \mathrm{U}(N) \otimes \mathrm{U}(N)$, where we generalise to $N$ degenerate flavors.

Because there is no chiral anomaly in $2+1 d$, it is possible to perform a change of variables in the path integral to identify two further "twisted" mass terms which, though antihermitian in Euclidian metric, are physically equivalent to the $m \bar{\psi} \psi$ of (1.1):

$$
\operatorname{im}_{3} \bar{\psi} \gamma_{3} \psi ; \quad i m_{5} \bar{\psi} \gamma_{5} \psi
$$

The "Haldane" mass term $m_{35} \bar{\psi} \gamma_{3} \gamma_{5} \psi$ is not equivalent because it changes sign under parity, and will not be considered further.

\section{The Thirring model in $2+1 d$}

A model of particular interest is the Thirring model, which has a contact interaction between conserved fermion currents. Its Lagrangian density reads

$$
\mathscr{L}=\bar{\psi}_{i}(\not \partial+m) \psi_{i}+\frac{g^{2}}{2 N}\left(\bar{\psi}_{i} \gamma_{\mu} \psi_{i}\right)^{2} ; i=1, \ldots, N
$$

Equivalently, its dynamics is captured by a bosonised action via the introduction of an auxiliary vector field $A_{\mu}$ :

$$
\mathscr{L}_{A}=\bar{\psi}_{i}\left(\not \partial+i \frac{g}{\sqrt{ } N} A_{\mu} \gamma_{\mu}+m\right) \psi_{i}+\frac{1}{2} A_{\mu}^{2} .
$$

The Thirring model is arguably the simplest interacting theory of fermions requiring a computational approach. The coupling $g^{2}$ has dimension $2-d$, where $d$ is the dimension of spacetime, so 
a naive expansion in powers of $g^{2}$ is non-renormalisable for $d>2$. However, things look different after resummation. First introduce an additional Stückelberg scalar $\varphi$ so the bosonic term becomes $\frac{1}{2}\left(A_{\mu}-\partial_{\mu} \varphi\right)^{2}$, to identify a hidden local symmetry [6]

$$
\psi \mapsto e^{i \alpha} \psi ; A_{\mu} \mapsto A_{\mu}+\partial_{\mu} \alpha ; \varphi \mapsto \varphi+\alpha .
$$

This point of view strongly suggests the identification of $A_{\mu}$ as an abelian gauge field; the original Thirring model (2.2) corresponds to a unitary gauge $\varphi=0$. In Feynman gauge, to leading order in $1 / N$ the resummed vector propagator is now of the form $\left\langle A_{\mu}(k) A_{v}(-k)\right\rangle \propto \delta_{\mu v} / k^{d-2}$. An expansion in powers of $1 / N$ may now be developed by analogy with $\mathrm{QED}_{3}$, and is exactly renormalisable for $2<d<4$ [7].

The outstanding theoretical issue is whether, for $g^{2}$ sufficiently large and $N$ sufficiently small, there is a symmetry-breaking transition leading to formation of a bilinear condensate $\langle\bar{\psi} \psi\rangle \neq 0$ accompanied by dynamical fermion mass generation. It is of particular interest to identify the critical $N_{c}$ above which symmetry breaking does not occur even in the strong coupling limit. The transitions at $g_{c}^{2}\left(N<N_{c}\right)$ then potentially define a series of distinct quantum critical points (QCPs). One early prediction, using strong-coupling Schwinger-Dyson equations in the ladder approximation, found $N_{c} \simeq 4.32$ [6].

\section{The Thirring model with staggered fermions}

The Thirring model has been studied by numerical simulations using staggered fermions, with action [8]

$$
S_{\text {stag }}=\frac{1}{2} \sum_{x \mu i}\left[\bar{\chi}_{x}^{i} \eta_{\mu x}\left(1+i A_{\mu x}\right) \chi_{x+\hat{\mu}}^{i}-\text { h.c }\right]+m \sum_{x i} \bar{\chi}_{x}^{i} \chi_{x}^{i}+\frac{N}{4 g^{2}} A_{\mu x}^{2} .
$$

Eq. (3.1) is not unique, but has the feature that the linear coupling of the auxiliary $A_{\mu}$ precludes higher-point interactions between fermions once it is integrated out. The action (3.1) has a global $\mathrm{U}(N) \otimes \mathrm{U}(N)$ symmetry broken to $\mathrm{U}(N)$ by either explicit or spontaneous mass generation. In a weakly coupled long-wavelength limit a $\mathrm{U}\left(2 N_{f}\right)$ symmetry is recovered with $N_{f}=2 N$ [9]; however the putative QCP is not weakly coupled, so this conventional wisdom must be questioned.

The action (3.1) has been studied for $N_{f} \in[2,6][8,10,11]$ and QCPs have been identified and characterised. Other studies with $N_{f}=2$ have found compatible results [12, 13]. A more recent study which identifies the strong-coupling limit reported $N_{f c}=6.6(1), \delta\left(N_{f c}\right) \approx 7$ [14]. The results are summarised in Fig. 1. The "chiral" symmetry is indeed broken for small $N_{f}$ and large $g^{2}$, and the exponent $\delta$ defined by the critical scaling $\left.\langle\bar{\chi} \chi\rangle\right|_{g_{c}^{2}} \propto m^{\frac{1}{\delta}}$ is very sensitive to $N_{f}$. Other exponents can be estimated using hyperscaling. These results are in qualitative but not quantitative agreement with the Schwinger-Dyson approach, which predicts $\delta\left(N_{f c}\right)=1$. A non-covariant form of (3.1) has been used to model the semimetal-insulator transition in graphene, finding $N_{f_{c}} \approx 5$ [15] suggesting that a Mott insulating phase is possible for the $N_{f}=2$ appropriate for monolayer graphene [16].

The staggered Thirring model thus exhibits a non-trivial phase diagram, with a sequence of QCPs with an $N$-dependence quite distinct from those of the theoretically much better-understood Gross-Neveu (GN) model. However, recent simulations of both models with $N_{f}=2$, using a fermion bag algorithm which permits study directly in the massless limit, have found compatible 


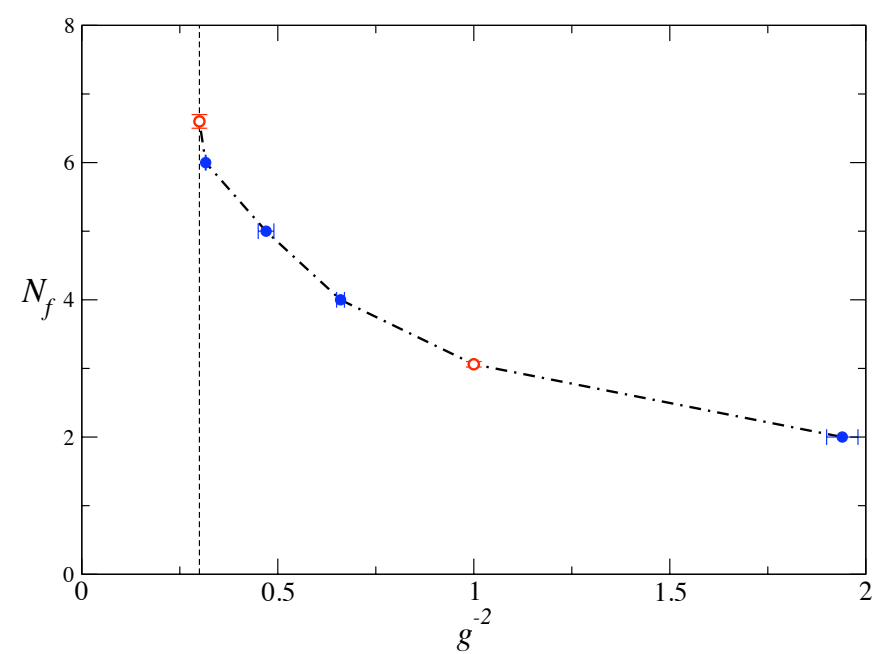

Figure 1: Phase diagram for the Thirring model with staggered fermions. A bilinear condensate is spontaneously generated in the region at lower left. The vertical dashed line denotes the effective strong coupling limit [14].

exponents for the QCP $[13,17]$ :

$$
\begin{aligned}
& v=0.85(1) ; \quad \eta=0.65(1) ; \quad \eta_{\psi}=0.37(1) ; \quad \text { Thirring } \\
& v=0.849(8) ; \quad \eta=0.633(8) ; \quad \eta_{\psi}=0.373(3) ; \quad \text { GN }
\end{aligned}
$$

The large- $N$ GN values are $v=\eta=1$. These results are troubling: from the perspective of the large- $N$ expansion using bosonised actions the models should be distinct, whereas (3.2) suggest rather they lie in the same RG basin of attraction. Indeed, when written purely in terms of four-point interactions between staggered fields spread over elementary cubes, the only difference between the models is an extra body-diagonal coupling in the GN case [17].

In this study we examine the possibility that staggered fermions do not reproduce the expected physics because of a failure to capture the correct continuum global symmetries near a QCP. A similar insight has been offered by the Jena group [18, 19, 20].

\section{Domain wall fermions in $2+1 d$}

The physical idea of domain wall fermions (DWF) is that fermions $\Psi, \bar{\Psi}$ are allowed to propagate along an extra fictitious dimension of extent $L_{s}$ with open boundary conditions. In $2+1+1 d$ this propagation is governed by an operator $\sim \partial_{3} \gamma_{3}$. As $L_{S} \rightarrow \infty$ zero modes of $D_{D W F}$ localised on the domain walls at either end become \pm eigenmodes of $\gamma_{3}$, and physical fields in the target $2+1 d$ space identified via

$$
\psi(x)=P_{-} \Psi(x, 1)+P_{+} \Psi\left(x, L_{s}\right) ; \quad \bar{\psi}(x)=\bar{\Psi}\left(x, L_{s}\right) P_{-}+\bar{\Psi}(x, 1) P_{+},
$$

with $P_{ \pm}=\frac{1}{2}\left(1 \pm \gamma_{3}\right)$. The walls are then coupled with a term proportional to the explicit mass $m$.

However, the emergence of the $\mathrm{U}(2 N)$ symmetry outlined in Sec. 1 is not manifest, because while the wall modes are eigenmodes of $\gamma_{3}$, the continuum symmetry $(1.2,1.3)$ demands equivalence under rotations generated by both $\gamma_{3}$ and $\gamma_{5}$. Another way of seeing this is that the twisted 
mass terms (1.4) should yield identical physics, eg. the strength of the corresponding bilinear condensate, as $L_{s} \rightarrow \infty$. This requirement is apparently non-trivial, since while $m$ and $m_{3}$ couple $\Psi$ and $\bar{\Psi}$ fields on opposite walls, $m_{5}$ couples fields on the same wall.

The recovery of $\mathrm{U}(2 N)$ symmetry as $L_{s} \rightarrow \infty$ was demonstrated numerically in a study of quenched non-compact $\mathrm{QED}_{3}$ on $24^{3} \times L_{s}$ systems, for a range of couplings $\beta$ [21]. First define the principal residual $\delta_{h}$ via the imaginary part of the twisted condensate:

$$
\mathfrak{I}\left[i\left\langle\bar{\Psi}(1) \gamma_{3} \Psi\left(L_{s}\right)\right\rangle\right]=-\mathfrak{I}\left[i\left\langle\bar{\Psi}\left(L_{s}\right) \gamma_{3} \Psi(1)\right\rangle\right] \equiv \delta_{h}\left(L_{s}\right) .
$$

The difference between the various condensates and their value in the large- $L_{S}$ limit is then specified in terms of secondary residuals $\varepsilon_{i}\left(L_{s}\right)$ via

$$
\begin{aligned}
\langle\bar{\psi} \psi\rangle_{L_{s}} & =i\left\langle\bar{\psi} \gamma_{3} \psi\right\rangle_{L_{s} \rightarrow \infty}+2 \delta_{h}\left(L_{s}\right)+2 \varepsilon_{h}\left(L_{s}\right) \\
i\left\langle\bar{\psi} \gamma_{3} \psi\right\rangle_{L_{s}} & =i\left\langle\bar{\psi} \gamma_{3} \psi\right\rangle_{L_{s} \rightarrow \infty}+2 \varepsilon_{3}\left(L_{s}\right) ; \\
i\left\langle\bar{\psi} \gamma_{5} \psi\right\rangle_{L_{s}} & =i\left\langle\bar{\psi} \gamma_{3} \psi\right\rangle_{L_{s} \rightarrow \infty}+2 \varepsilon_{5}\left(L_{s}\right) .
\end{aligned}
$$

Empirically, as shown in Fig. 2, the residuals $\delta_{h}$ and $\varepsilon_{i}$ decay exponentially with $L_{S}$, with a clear

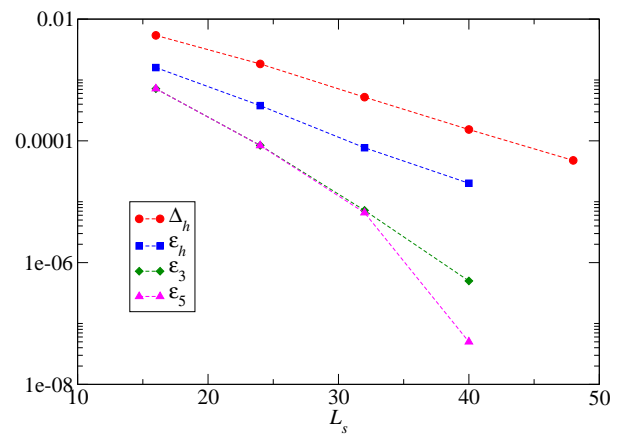

Figure 2: Residuals $\delta_{h}, \varepsilon_{i}$ as functions of $L_{s}$ in quenched non-compact $\mathrm{QED}_{3}$.

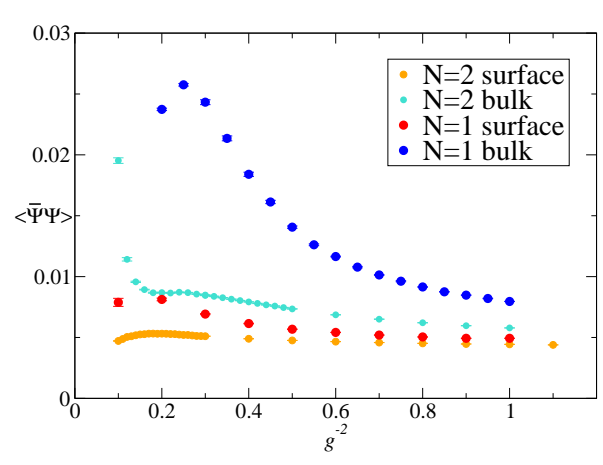

Figure 3: $i\left\langle\bar{\psi} \gamma_{3} \psi\right\rangle$ vs. $g^{-2}$ for both surface and bulk Thirring models.

hierarchy $\delta_{h} \gg \varepsilon_{h} \gg \varepsilon_{3} \equiv \varepsilon_{5}$. This is evidence for the ultimate recovery of $\mathrm{U}(2 N)$ (the convergence rate is strongly dependent on both $\beta$ and system volume), and moreover suggests the optimal simulation strategy is to focus on the twisted condensate $i\left\langle\bar{\psi} \gamma_{3} \psi\right\rangle$ for which finite- $L_{s}$ corrections are minimal. The equivalence of $\gamma_{3}$ and $\gamma_{5}$ condensates at finite $L_{s}$ and their superior convergence to the large- $L_{s}$ limit was shown analytically in [22], where the convergence to fermions obeying $2+1 d$ Ginsparg-Wilson relations was also demonstrated. Exponential improvement of convergence was demonstrated in the large- $N$ limit of the GN model in [23]. The benefits of a twisted mass term for improved recovery of $\mathrm{U}(2 N)$ symmetry were also observed in a study of non-compact $\mathrm{QED}_{3}$ using Wilson fermions in [24].

\section{The Thirring model with domain wall fermions}

Even after settling on DWF, we still encounter some remaining formulational issues, reviewed more thoroughly in $[23,25]$. Just as for the staggered model, we have chosen a linear interaction 
between the fermion current and a $2+1 d$ vector auxiliary field $A_{\mu}$ defined on the lattice links. The simplest approach to formulating the Thirring model is to restrict the interaction to the physical fields (4.1) defined on the domain walls at $s=1, L_{s}$. This follows the treatment of the GN model with DWF developed in [26]. It has the technical advantage that the Pauli-Villars fields required to cancel bulk mode contributions to the fermion determinant do not couple to $A_{\mu}$, and hence can be safely excluded from the simulation, which brings significant cost savings. In what follows this approach will be referred to as the Surface model.

However, following the discussion below eq. (2.3) suggesting the similarity of $A_{\mu}$ with an abelian gauge field, we also consider a Bulk formulation in which $\Psi, \bar{\Psi}$ interact with a "static" field (ie. $\partial_{3} A_{\mu}=0$ ) throughout the bulk:

$$
\mathscr{S}=\bar{\Psi} \mathscr{D} \Psi=\bar{\Psi} D_{W} \Psi+\bar{\Psi} D_{3} \Psi+m_{i} S_{i}
$$

with

$$
D_{W}=\gamma_{\mu} D_{\mu}-\left(\hat{D}^{2}+M\right) ; \quad D_{3}=\gamma_{3} \partial_{3}-\hat{\partial}_{3}^{2}
$$

and $m_{i} S_{i}$ is the explicit mass term defined only on the walls. Here

$$
\begin{aligned}
D_{\mu x y} & =\frac{1}{2}\left[\left(1+i A_{\mu x}\right) \delta_{x+\hat{\mu}, y}-\left(1-i A_{\mu x-\hat{\mu}}\right) \delta_{x-\hat{\mu}, y}\right], \\
\hat{D}_{x y}^{2} & =\frac{1}{2} \sum_{\mu}\left[\left(1+i A_{\mu x}\right) \delta_{x+\hat{\mu}, y}+\left(1-i A_{\mu x-\hat{\mu}}\right) \delta_{x-\hat{\mu}, y}-2 \delta_{x y}\right],
\end{aligned}
$$

and $M$ is the domain wall height, here set equal to $1 . \partial_{3}, \hat{\partial}_{3}^{2}$ are defined similarly using finite differences in the 3 direction, respecting the open boundary conditions, and with no coupling to the auxiliary. These definitions imply the following properties:

$$
\left[\partial_{3}, D_{\mu}\right]=\left[\partial_{3}, \hat{D}^{2}\right]=0 \text { but }\left[\partial_{3}, \hat{\partial}_{3}^{2}\right] \neq 0 \text { on domain walls. }
$$

The action (5.1) may be simulated using the HMC algorithm for $N$ even; however the failure of the third commutator in (5.4) to vanish everywhere is an obstruction to proving $\operatorname{det} \mathscr{D}$ is positive definite, so $N=1$ is simulated using the RHMC algorithm with functional measure $\operatorname{det}\left(\mathscr{D}^{\dagger} \mathscr{D}\right)^{\frac{1}{2}}$.

\section{Numerical results}

We have studied both surface and bulk formulations of the Thirring model in the coupling range $a g^{-2} \in[0.1,1.0]$ with first $N=2$ [23] and now $N=1$ [25]. The RHMC algorithm uses 25 partial fractions to estimate fractional powers of $\mathscr{D}^{\dagger} \mathscr{D}$. Most results are obtained on $12^{3} \times 16$ $(N=2)$ or $12^{3} \times 8(N=1)$. Summary results for the bilinear condensate $i\left\langle\bar{\psi} \gamma \gamma_{3} \psi\right\rangle$ with $m_{3} a=0.01$ are shown in Fig. 3. The bulk model shows enhanced pairing compared to the surface model, and as might be anticipated pairing is greater for $N=1$ than $N=2$. For $N=1 i\left\langle\bar{\psi} \gamma_{3} \psi\right\rangle$ rises sharply for $a g^{-2} \lesssim 0.5$, until a maximum at $a g^{-2} \approx 0.25$. In previous work with staggered fermions this maximum has been identified with the effective location of the continuum strong coupling limit [14], since for stronger lattice couplings there is a breakdown of reflection positivity [8]. We are thus confident that the range of couplings explored includes the strong coupling limit. 
Fig. 4 shows the auxiliary action over the same coupling range, compared with the free field value $\frac{d}{2}$. The difference between surface and bulk models is apparently very striking, but remember this is really a comparison of UV properties of two different regularisations of ostensibly the same continuum field theory. Once again, there is tentative evidence for a change of behaviour of the $N=1$ bulk model at $a g^{-2} \approx 0.5$.

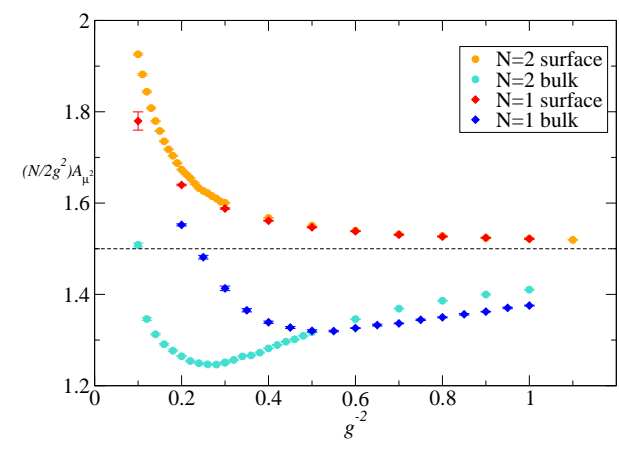

Figure 4: Auxiliary action vs. $g^{-2}$.

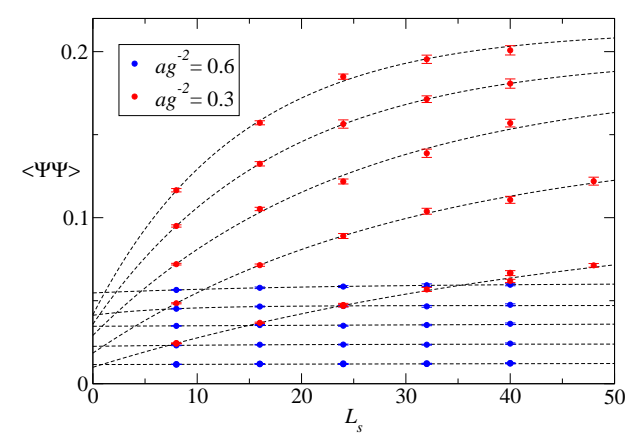

Figure 5: $\left\langle\bar{\psi} i \gamma_{3} \psi\left(L_{S}\right)\right\rangle$ on $12^{3}$ for $a g^{-2}=0.3,0.6$.

In order to address the issue of $\mathrm{U}(2)$ symmetry breaking it is necessary to take the limit $L_{s} \rightarrow \infty$. We have performed RHMC simulations on $12^{3} \times L_{s}$ systems with $L_{s}$ ranging up to 48 [25]. Fig 5 shows how the condensate varies with $L_{s}$ at two representative couplings, $a g^{-2}=0.6$ (weak) and $a g^{-2}=0.3$ (strong), and for masses $m a=0.01, \ldots, 0.05$. The finite volume corrections are more significant at strong coupling. Empirically, they are well-described by the 3-parameter form

$$
\langle\bar{\psi} \psi\rangle_{L_{s}=\infty}-\langle\bar{\psi} \psi\rangle_{L_{s}}=2 \varepsilon_{3}\left(L_{s}, m, g^{2}\right)=A\left(m, g^{2}\right) e^{-\Delta\left(m, g^{2}\right) L_{s}}
$$

The Ansatz (6.1) was trialled for data taken in the quenched approximation [25], where it was found that $L_{s} \rightarrow \infty$ must be taken before $m \rightarrow 0$. Extrapolated condensate data for $N=1$ are plotted in Fig. 6.

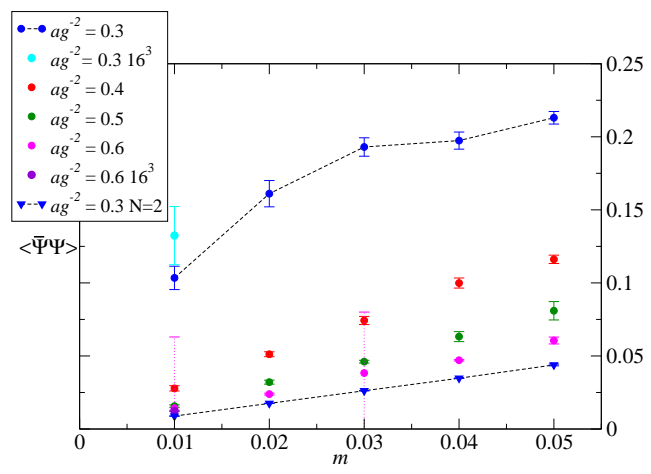

Figure 6: $\left\langle\bar{\psi} i \gamma_{3} \psi\left(m_{3}\right)\right\rangle$ for $N=1$ in the $L_{S} \rightarrow \infty$ limit.

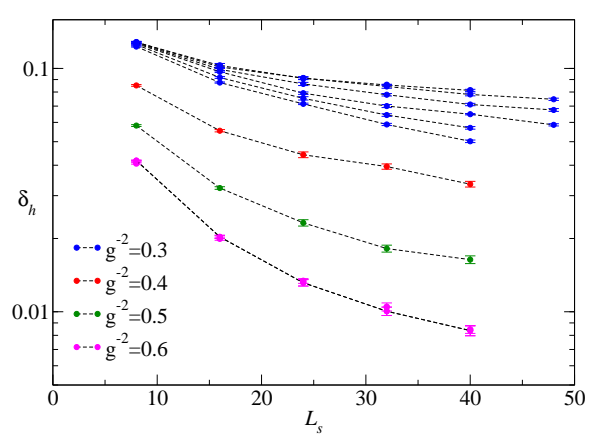

Figure 7: The residual $\delta_{h}\left(L_{S}\right)$ quantifying approach to the $\mathrm{U}(2)$-symmetric limit. 
Fig. 6 shows that for $a g^{-2} \gtrsim 0.4$ the condensate is a linear function of $m$ intercepting the origin as $m \rightarrow 0$; in other words the bilinear condensate vanishes in the massless limit and $\mathrm{U}(2)$ remains unbroken. The same is true for $N=2$ data extrapolated to $L_{s} \rightarrow \infty$ at the strongest available coupling, confirming the conclusion of [23] that for $N=2 \mathrm{U}(2)$ is unbroken for all couplings. By contrast for the strongest coupling $a g^{-2}=0.3$ explored for $N=1$, the condensate is at least a factor of two greater, exhibits significant finite volume effects at small $m$, and appears to support a nonzero intercept as $m \rightarrow 0$; here the $\mathrm{U}(2)$ is broken. The most compelling interpretation of the data in Fig. 6 is thus

$$
1<N_{c}<2 .
$$

Finally, Fig. 7 plots the residual $\delta_{h}\left(g^{2}, m ; L_{s}\right)$ defined in (4.2). The data is consistent with asymptotic behaviour $\delta_{h} \propto e^{-c L_{s}}$, implying $\mathrm{U}(2)$ restoration in the large- $L_{s}$ limit, with the constant $c m$-independent at weak coupling but strongly $m$-dependent in the proposed broken symmetry phase at $a g^{-2}=0.3$, seen in Fig. 7 where the blue points fan out. The restoration of U(2) looks very slow, which may present practical difficulties in future studies of the broken phase. No-one promised simulations at a strong coupling fixed-point would be easy...

\section{Summary and outlook}

It has been shown that it is feasible to use DWF to study $\mathrm{U}(2 N)$-symmetric fermions in $2+1 d$, and that use of a twisted mass term $\sim i m_{3} \bar{\psi} \gamma_{3} \psi$ optimises the recovery of the symmetry as $L_{s} \rightarrow \infty$. A study of the Thirring model at strong fermion self-couplings then shows that DWF capture a very different physics to that described by staggered fermions, which are governed by a different global symmetry away from the weak-coupling long-wavelength limit. While there is no evidence for symmetry breaking with $N=2$, the latest results obtained in the large- $L_{s}$ limit for $N=1$ using RHMC suggest that a broken symmetry phase is present at strong coupling, and hence $1<N_{c}<2$. Note a disparity between DWF and staggered fermions in a very different physical context, namely near a conformal fixed point in a $3+1 d$ non-abelian gauge theory, has also been reported [27]. Away from weak coupling, a faithful rendition of global symmetries appears to be crucial.

In ongoing and future work we plan to return to the issues of particle spectroscopy, and an assessment of $\mathrm{U}(2)$ symmetry recovery via the axial Ward identity; exploratory results for both were presented in [23], and an update appears in [25]. At some point it will also be necessary to study the locality properies of the corresponding $2+1 d$ overlap operator, furnishing a non-trivial test of the DWF approach to relativistic fermions in a new physical context. Additionally, functional renormalisation group studies indicate that in the hunt for a QCP it may be interesting to include a $\mathrm{U}(2 N)$-invariant Haldane interaction $-\tilde{g}^{2}\left(\bar{\psi} \gamma_{3} \gamma_{5} \psi\right)^{2}$ [28]; the control offered by DWF will make this a straightforward exercise.

\section{References}

[1] Z. Tešanović, O. Vafek, M. Franz, Phys. Rev. B65, 180511 (2002)

[2] I.F. Herbut, Phys. Rev. B66, 094504 (2002), cond-mat / 0202491

[3] X.G. Wen, Phys. Rev. B65, 165113 (2002) 
[4] W. Rantner, X.G. Wen, Phys. Rev. B66, 144501 (2002)

[5] A.H. Castro Neto, F. Guinea, N.M.R. Peres, K.S. Novoselov, A.K. Geim, Rev. Mod. Phys. 81, 109 (2009), 0709.1163

[6] T. Itoh, Y. Kim, M. Sugiura, K. Yamawaki, Prog. Theor. Phys. 93, 417 (1995), hep-th/ 9411201

[7] S. Hands, Phys. Rev. D51, 5816 (1995), hep-th/9411016

[8] L. Del Debbio, S.J. Hands, J.C. Mehegan (UKQCD), Nucl. Phys. B502, 269 (1997), hep-lat/9701016

[9] C. Burden, A.N. Burkitt, Europhys. Lett. 3, 545 (1987)

[10] L. Del Debbio, S.J. Hands, Nucl. Phys. B552, 339 (1999), hep-lat/ 9902014

[11] S. Hands, B. Lucini, Phys. Lett. B461, 263 (1999), hep-lat/9906008

[12] E. Focht, J. Jersák, J. Paul, Phys. Rev. D53, 4616 (1996), hep-lat / 9511005

[13] S. Chandrasekharan, A. Li, Phys. Rev. Lett. 108, 140404 (2012), 1111 . 7204

[14] S. Christofi, S. Hands, C. Strouthos, Phys. Rev. D75, 101701 (2007), hep-lat/ 0701016

[15] S. Hands, C. Strouthos, Phys. Rev. B78, 165423 (2008), 0806.4877

[16] W. Armour, S. Hands, C. Strouthos, Phys. Rev. B81, 125105 (2010), 0910.5646

[17] S. Chandrasekharan, A. Li, Phys. Rev. D88, 021701 (2013), 1304.7761

[18] D. Schmidt, B. Wellegehausen, A. Wipf, PoS LATTICE2015, 050 (2016), 1511.00522

[19] D. Schmidt, B. Wellegehausen, A. Wipf, PoS LATTICE2016, 247 (2016), 1611.00275

[20] B.H. Wellegehausen, D. Schmidt, A. Wipf, Phys. Rev. D96, 094504 (2017) 1708.01160

[21] S. Hands, JHEP 09, 047 (2015), 1507 . 07717

[22] S. Hands, Phys. Lett. B754, 264 (2016), 1512.05885

[23] S. Hands, JHEP 11, 015 (2016), 1610.04394

[24] N. Karthik, R. Narayanan, Phys. Rev. D93, 045020 (2016), 1512.02993

[25] S. Hands, On the Critical Flavor Number in the 2+1d Thirring Model, 1811.04818

[26] P. Vranas, I. Tziligakis, J.B. Kogut, Phys. Rev. D62, 054507 (2000), hep-lat/ 9905018

[27] A. Hasenfratz, C. Rebbi, O. Wetzel, EPJ Web Conf. 175, 03006 (2018), 1708 . 03385

[28] F. Gehring, H. Gies, L. Janssen, Phys. Rev. D92, 085046 (2015), 1506.07570 